\title{
Role of defects in the onset of wall-induced granular convection
}

\author{
Andrea Fortini ${ }^{1,2, *}$ and Kai Huang ${ }^{3}$ \\ ${ }^{1}$ Theoretische Physik II, Physikalisches Institut, Universität Bayreuth, Universitätsstraße 30, D-95447 Bayreuth, Germany \\ ${ }^{2}$ Department of Physics, University of Surrey, Guildford GU2 7XH, United Kingdom \\ ${ }^{3}$ Experimentalphysik V, Physikalisches Institut, Universität Bayreuth, Universitätsstraße 30, D-95447 Bayreuth, Germany
}

(Received 2 June 2014; revised manuscript received 9 February 2015; published 27 March 2015)

\begin{abstract}
We investigate the onset of wall-induced convection in vertically vibrated granular matter by means of experiments and two-dimensional computer simulations. In both simulations and experiments we find that the wall-induced convection occurs inside the bouncing bed region of the parameter space, in which the granular bed behaves like a bouncing ball. A good agreement between experiments and simulations is found for the peak vibration acceleration at which convection starts. By comparing the results of simulations initialized with and without defects, we find that the onset of convection occurs at lower vibration strengths in the presence of defects. Furthermore, we find that the convection of granular particles initialized in a perfect hexagonal lattice is related to the nucleation of defects and the process is described by an Arrhenius law.
\end{abstract}

DOI: 10.1103/PhysRevE.91.032206

PACS number(s): 45.70.-n, 61.72.Bb, 47.55.P-

\section{INTRODUCTION}

Mixing and demixing of vibrated granular matter [1,2] are of importance in nature [3] as well as in many industrial processes. For example, they are used in the pharmaceutical, construction [4], and waste reprocessing [5] industries. The term Brazil nut effect (BNE) [6], which originally referred to the rise of a large particle to the top of a container filled with smaller grains, is now used to indicate the more general demixing of differently sized particles under vertical oscillations. Schröter et al. [7] reviewed and identified seven possible mechanisms that lead to the BNE and to the reverse Brazil nut ffect (RBNE) [8]. Convective cells induced by the walls of the container were found to be a major contributing mechanism to the occurrence of the BNE [9-13].

The complexity of the BNE characterization is in part due to an underlying dynamical behavior which even for one-component systems is very rich. At driving accelerations smaller than the gravitational acceleration, the granular bed comoves with the bottom wall. Upon increasing the acceleration the granular bed behaves like a bouncing ball [14], and above this bouncing bed region collective undulations (also known as arches) appear [15-18]. At still higher accelerations the granular Leidenfrost effect occurs, in which a dense granular fluid hovers over a granular gas. Recently, Eshuis et al. [19] systematically drew phase diagrams for all these phenomena, and found at very high accelerations a convective regime in which the sample is completely fluidized $[18,19]$.

However, this convection regime is distinct from the wall-induced convection which occurs at low accelerations and is one of the driving mechanisms for the BNE. The wall-induced convection is caused by the shear forces between particles and walls. During the upward acceleration the mixture gets compacted and shear forces induced by the side walls propagate efficiently through the whole sample. During the downward motion the mixture is more expanded and consequently those particles adjacent to the walls experience stronger downward shear forces than those in the center of the

*andrea.fortini@uni-bayreuth.de container. The combination of the two types of motion gives rise to convection [7]. This cycle of expansion and compression of the granular bed was studied by Sun et al. [20] and found to be strongly dependent on wall friction. Even though their study was done in relation to the BNE, no connection to the convective motion is made. The onset of convection has been studied extensively both in experiments [21,22] and with numerical simulations [23-26].

In this article, we study with both computer simulations and experiments the dynamical phase diagram of two-dimensional vertically oscillated granular matter and analyze the mechanism behind the onset of the wall-induced convection to clarify the role of topological defects.

\section{METHODS}

For the theoretical investigation we carry out molecular dynamics (MD) [27] simulations of a two-dimensional system in a box of size $L_{x} \times L_{z}$ delimited by flat hard walls and with gravity $\boldsymbol{g}=-\boldsymbol{g} \boldsymbol{e}_{z}$ pointing in the negative $z$ direction. The particles have two translational degrees of freedom in the $x$ and $z$ directions and one rotational degree of freedom about the perpendicular $y$ axis. The granular beads are described as soft disks of diameter $\sigma$, mass $m$, and moment of inertia $I=m \sigma^{2} / 8$, which interact via a linear contact model with viscoelastic damping between the disks and via static friction [28]. This model and its parameter values (see Table I) have been chosen because they reproduce the contact properties [29] of granular beads, and give results for the dynamics of an intruder in a vertically oscillated granular bed that are in good agreement with experiments [20]. The simulation box is driven sinusoidally, i.e., the bottom of the container is moved in time according to

$$
z_{b}=-\frac{1}{2} L_{z}+A \sin (\omega t),
$$

where $z_{b}$ is the height coordinate of the bottom of the container, $A$ is the amplitude of the oscillation, $\omega$ is the frequency, and $t$ is the time.

We traced the dynamical phase diagram for a fixed oscillation frequency $\omega=1.0 t_{0}^{-1}$ and lateral wall separation $L_{x} / \sigma=20$. Reduced units are used throughout the article: the 
TABLE I. Numerical values of the simulation parameters.

\begin{tabular}{lccc}
\hline \hline Coefficient & & Particle-particle & Wall-particle \\
\hline Normal stiffness & $k_{n}$ & $10^{6}\left(k_{0}\right)$ & $10^{6}\left(k_{0}\right)$ \\
Tangential stiffness & $k_{t}$ & $10^{4}\left(k_{0}\right)$ & $10^{4}\left(k_{0}\right)$ \\
Static friction & $\mu$ & 0.6 & 0.6 \\
Normal damping & $\gamma_{n}$ & $100\left(\gamma_{0}\right)$ & $100\left(\gamma_{0}\right)$ \\
Tangential damping & $\gamma_{t}$ & $100\left(\gamma_{0}\right)$ & $100\left(\gamma_{0}\right)$ \\
\hline \hline
\end{tabular}

particle mass $m$, the particle diameter $\sigma$, and the gravitational acceleration $g$ are our fundamental units. Consequently, the derived units are the time $t_{0}=\sqrt{\sigma / g}$, velocity $v_{0}=\sqrt{g \sigma}$, force $f_{0}=m g$, elastic constant $k_{0}=m g / \sigma$, and damping coefficient $\gamma_{0}=\sqrt{g / \sigma}$. Further details of the model are given in Appendix A.

The external driving force is characterized by a dimensionless acceleration $\Gamma=A \omega^{2} / g$, corresponding to the maximum acceleration due to Eq. (1) divided by the gravitational acceleration $g$. Alternatively, we use the dimensionless energy parameter [30] $K_{m}=\frac{A^{2} \omega^{2}}{\sigma g}$, i.e., the maximum kinetic energy per particle "injected" into the system at every period of oscillation [31].

The experiment is conducted with a monolayer of spherical polished opaque glass beads ( $\mathrm{SiLiBeads} \mathrm{P}$ ) with a diameter of $2 \pm 0.02 \mathrm{~mm}$. A rectangular cell made up of two glass plates of $40 \mathrm{~mm}$ width by $200 \mathrm{~mm}$ height separated by a distance of $2.3 \mathrm{~mm}$ is used to create a quasi-two-dimensional configuration. The cell is mounted on an electromagnetic shaker (Tira TV50350) with the sinusoidal frequency and amplitude controlled by a function generator (Agilent FG33220). The acceleration is obtained by an accelerometer (Dyson 3035B2). In order to avoid influence from electrostatic forces, the side walls of the container are made of aluminum. With backlight illumination, the mobility of the particles is captured with a high-speed camera (IDT MotionScope M3) mounted in front of the cell. The camera is externally triggered so as to take images at fixed phases of each vibration cycle. The snapshots captured are subjected to an image processing procedure to locate all spheres based on a Hough transformation [32]. Tracer particles are used to determine the thresholds for the bouncing bed phase and for the start of convection (see Appendix B for details).

In order to compare the results of the simulations with the experiments, we produced results for fixed numbers of particles, namely $N=200,400,800,1200,1600$. The different sets are identified via the linear density $N_{l}=N / L_{x}$. This number gives an approximate value for the number of particle layers and hence the height of the granular bed. In reality the height depends on the local structure of the granular bed, namely, the orientation of the hexagonally ordered particles, and the type and amount of defects.

\section{INITIALIZATION}

In the experiment the system is initialized with a strong agitation to create a completely fluidized state, followed by a slow ramping down of the vertical acceleration. In the simulation the initial configuration is prepared with two
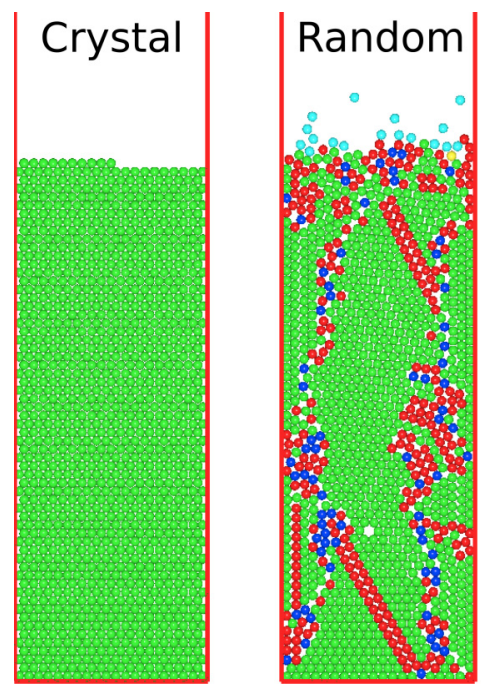

\section{Experiment}

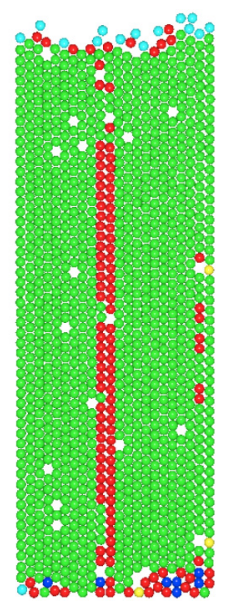

FIG. 1. (Color online) Simulation snapshots of the typical initial configurations for $N_{l}=60$. The color indicates the local order of the particles according to an analysis with the $q_{6}$ order parameter [33]. Green (light gray) indicates hexagonal order and red (dark gray) indicates a square local order while other colors indicate a disordered configuration. From left to right: the crystalline and random initializations of the simulation, and the experimental initial configuration.

distinct procedures. In the crystal initialization, the particles are placed in a perfect hexagonal lattice resting at the bottom of the container. In the random initialization, the particles are placed randomly in the box. Via molecular dynamics we evolve the system until all particles have fallen under gravity and have reached a rest position.

The resulting configurations are shown in Fig. 1. We note that in both simulations initialized randomly and the experiments a large amount of particles have local hexagonal order with many topological defects, such as dislocations and grain boundaries. For a two-dimensional system there are two favored orientations of the hexagonal lattice, when in contact with a flat wall. In Fig. 2(a) the orientation A with the [111] direction parallel to the wall is shown. In Fig. 2(b) the orientation B has the [010] direction parallel to the wall. In the crystalline initialization we chose to place the particles according to orientation $\mathrm{A}$. After the random initialization (a)

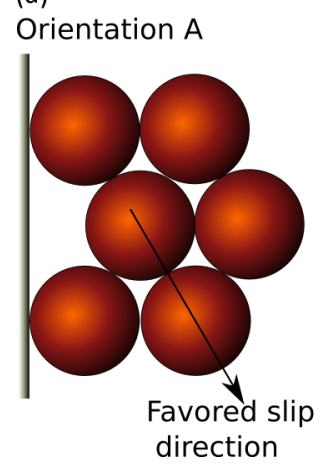

(b)

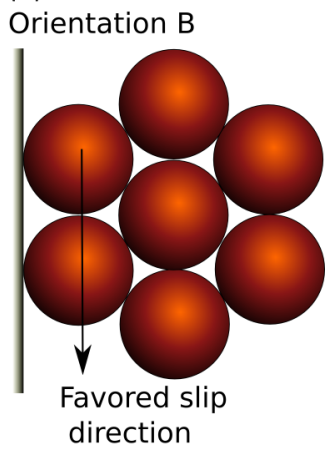

FIG. 2. (Color online) Sketch of the possible orientations of the hexagonal crystal in contact with the lateral wall of the container box. 
processes, we find that in the experiment almost all particles have orientation $\mathrm{B}$, while in simulations the orientation $\mathrm{A}$ seems to be favored, but grain boundaries between the different orientations are visible. Figure 1 shows the configurations obtained in the three cases.

\section{DYNAMICAL PHASES}

We have systematically investigated the onset of convection at different dimensionless accelerations or energies and heights of the granular bed. Figure 3 shows the location of the dynamical phases as a function of the dimensionless acceleration $\Gamma$ and the energy parameter $K_{m}$ for different values of the linear density $N_{l}$. The parameter $N_{l} \sigma$ is also a measure of the number of layers in the granular bed, and therefore of its height.

The transition from the comoving to the bouncing bed phase occurs, as expected, at $\Gamma$ slightly larger than 1 , with the critical value of the acceleration increasing slightly with increasing number of layers. We find good agreement between simulations (green triangles and blue circles) and experiments (connected squares). In experiments, the determination of this boundary is sensitive to the accuracy of the particle position determination, which is set by the resolution of the camera.

Inside the bouncing bed regime, we observe a transition from a bouncing bed phase without convection to a bouncing bed phase with convection. We find reasonable agreement between experiments and the simulations initialized randomly. For $N_{l} \sigma=60,80$ the model underestimates the amount of energy necessary for the convection to start. The discrepancy is probably related to variability in the concentration of defects, as well as to the presence of the front and back walls in the experiments. For simulations that start without topological

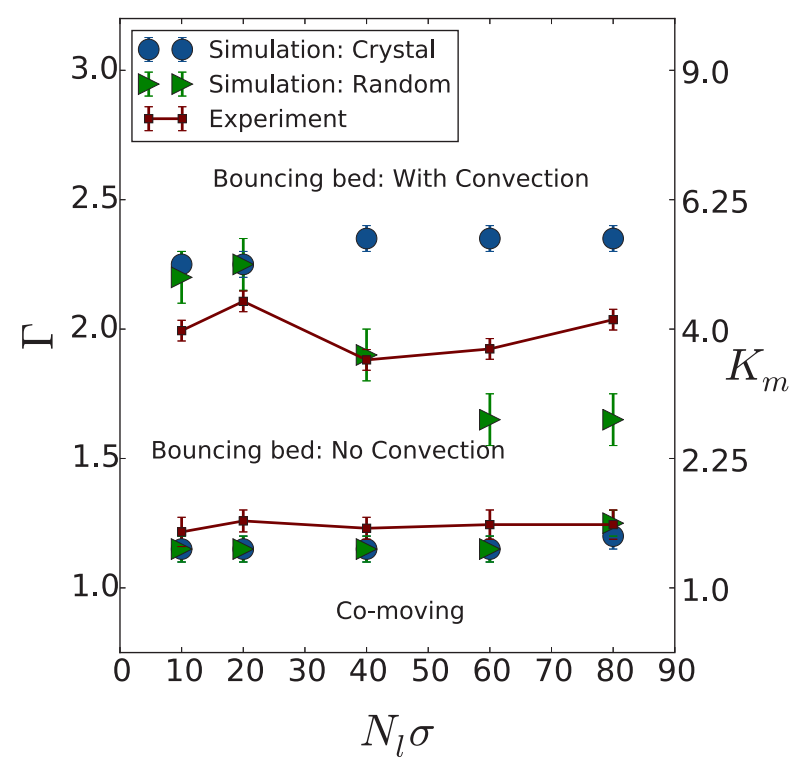

FIG. 3. (Color online) Dynamical phases at different accelerations $\Gamma$ and linear density (number of layers) $N_{l} \sigma$. On the right $y$ axis the energy parameter $K_{m}$ is also reported. Symbols are for simulation results for either the system initialized randomly (triangles) or in a crystalline configuration (circles). The connected squares are the experimental results. defects we consistently detect the onset of convection (blue circles) at higher values of the acceleration with respect to the experiments and to the simulations started with defects. The enhancement of the convection due to the presence of defects explains the observation of Pöschel and Herrmann [11] that an intruder can initiate convection. The presence of an intruder induces topological defects [34] that initiate the convection and segregation.

\section{ONSET OF CONVECTION}

In crystalline materials collective particle movements occur via crystalline plane slips [10]. The same occurs in our granular systems, but we noted some differences in slip behavior between simulation and experiments. In simulation the slips occur primarily along the oblique directions, while in the experiment they occur mainly along the vertical direction. The difference can be explained by the different orientation of the hexagonal crystal in the two cases. The granular particles experience a shear stress along the vertical direction due to the wall of the container. In the experiments the particles are oriented as in Fig. 2(b), and slips occur preferentially in the vertical direction. On the other hand, in simulations we find more particles with an orientation as in Fig. 2(a) and the pyramidal slip planes will be activated first. As long as plane slips are concerned, the worst case scenario is represented by a perfect crystal with orientation A. Since no defects are initially present, slips along the pyramidal planes can occur only if defects are nucleated first. Another difference is that in experiments we often observe single convective rolls similar to the observation in a two-dimensional rotating cell [35]. This type of convective motion is not detected in simulations and the reason for the discrepancy is likely due to a small tilt of the side walls [9].

In order to clarify the role of defects in the onset of convection, we analyze in computer simulations the granular temperature $T_{g}$ in relation to the average input energy $K_{m}$ The granular temperature is defined as

$$
T_{g}=\frac{m}{N}\left\langle\sum_{i=1}^{N} \frac{1}{2}\left[\boldsymbol{v}_{i}(t)-\boldsymbol{v}_{\mathrm{c} . \mathrm{m} .}(t)\right]^{2}\right\rangle,
$$

where $\boldsymbol{v}_{\text {c.m. }}$ is the center of mass velocity, $\boldsymbol{v}_{i}(t)$ is the velocity of particle $i$ at time $t$, and \langle\rangle indicates a time average. Figures 4(a) and 4(b) show the behavior of the reduced granular temperature $T_{g}^{*}=T_{g} /(m g \sigma)$ as a function of the dimensionless energy parameter $K_{m}$ for systems initialized with and without defects, respectively. In both cases the temperature increases monotonically over many orders of magnitude. The curve gives an indication of how much input energy $K_{m}$ is converted into the kinetic energy of the granular particles.

We divide the energy $K_{m}$ parameter space into regions with different slopes of the temperature curves. For the random initialization [Fig. 4(a)] we find two regions $A$ and $B$. The transition occurs at $K_{m} \simeq 3.3$ as signaled by a change in slope of the temperature curve. Comparing the energy value for this transition with the diagram of Fig. 3, we note that it corresponds to the energy value at the onset of convection. 

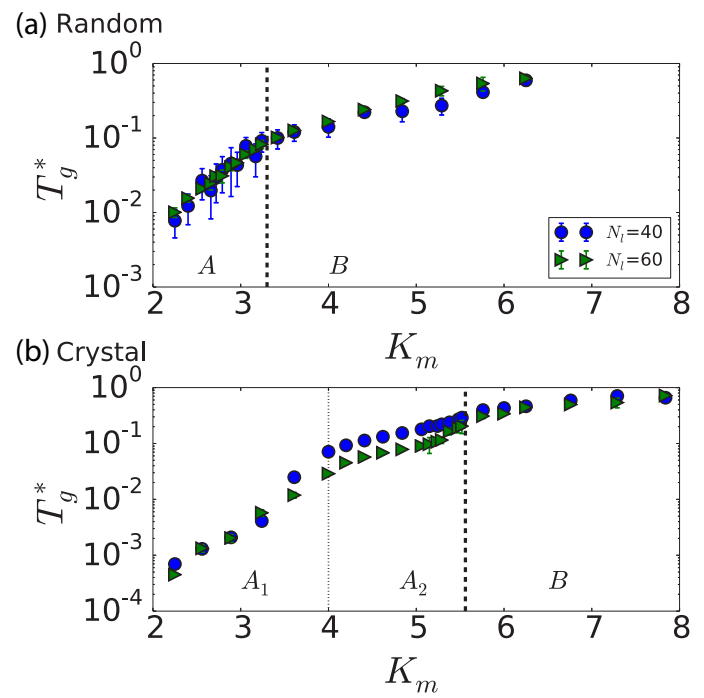

FIG. 4. (Color online) Granular temperature $T_{g}$ versus dimensionless energy parameter $K_{m}$. The dashed lines separate regions with different slopes of the temperature curves. (a) For the system initialized randomly, i.e., with defects. (b) For the system initialized in a perfect hexagonal lattice, i.e., without initial defects.

On the other hand, for the case initialized without defects [Fig. 4(b)] we can distinguish three regions. Following the naming convention used in Fig. 4(a), we indicate by $B$ the region where convection is detected. The region $A$, without convection, is now divided into two subregions $A_{1}$ and $A_{2}$.

In order to clarify the origin of the different regions of temperature behavior, we investigate the slip probability of a particle in the bulk of the granular bed, i.e., away from the top free interface [36]. The slip of a particle is detected if a displacement larger than $0.2 \sigma$ is measured for at least two nearest neighbors after one oscillation. We define the slip probability $p_{s}$ as the fraction of particles which undergo a slip in one oscillation period. The value of $p_{s}$ is averaged over 200 periods of oscillation. The slip probability as a function of $1 / K_{m}$ is shown in Figs. 5(a) and 5(b) for the random and crystal initializations, respectively. For the random case we do not observe a change in the slip behavior between regions $A$ and $B$. On the other hand a quite dramatic change of behavior is observed for the crystal case. In particular, in region $A_{1}$ the number of detected slips is exactly zero, while region $A_{2}$ is characterized by a quite steep increase of slip events, which slows down considerably upon the onset of convection (region $B$ ).

In all cases the curves can be fitted to an Arrhenius law $p_{s} \propto \exp \left(-E_{b} / K_{m}\right)$, indicating the presence of activating mechanisms. The energy parameter $E_{b}$ is a measure of the barrier height and the dimensionless energy $K_{m}$ assumes the role of a reservoir temperature. For the random case just a single curve can be fitted to the entire range of inverse energies. From the fit we find an average dimensionless barrier height $E_{b}=7 \pm 1$. For the case initialized with the crystal, we find a barrier $E_{b}=130 \pm 20$ in region $A_{2}$ and a barrier $E_{b}=7 \pm 1$ in region $B$.

Since the barrier height is the same for the system with initial defects, independent of the presence of convection, we (a) Random

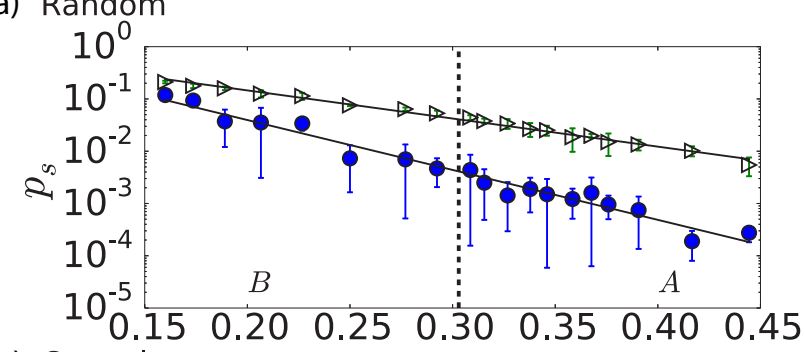

(b)

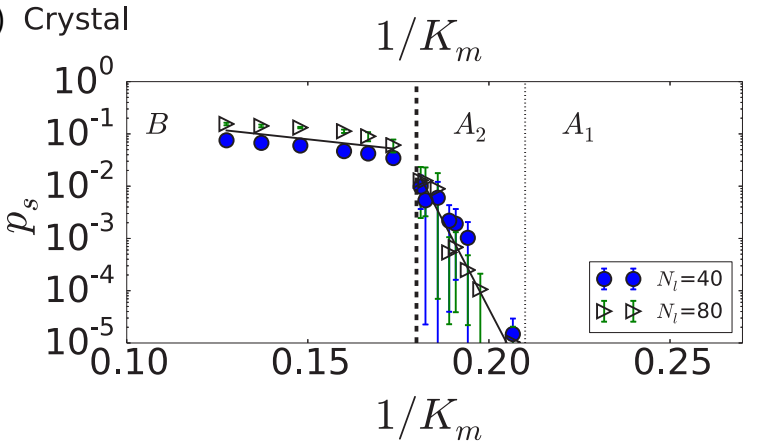

FIG. 5. (Color online) Slip probability $p_{s}$ versus the inverse dimensionless energy parameter $1 / K_{m}$. The continuous lines represent exponential fit functions. The dashed lines separate the regions defined in Fig. 4. (a) For the system initialized randomly, i.e., with defects. (b) For the system initialized in a perfect hexagonal lattice, i.e., without initial defects.

can speculate that the activation mechanism indicated by the Arrhenius equation is the activation of slip events in a crystal with topological defects. Interestingly, the same barrier is measured for the system without initial defects in region $B$, suggesting the same activation mechanism. But in order for this to occur defects must first nucleate inside a perfect crystal, and we speculate that the very high barrier in region $A_{2}$ is due to the nucleation of defects in a perfect hexagonal crystal.

\section{CONCLUSIONS}

In conclusion, using two-dimensional computer simulations and experiments we locate the wall-induced convection in the bouncing bed region in the dynamical phase diagram of vertically vibrated granular matter. For the onset of convection, we find a reasonable agreement between the experimental results and those from simulations initialized with defects. We believe the reason behind the discrepancy is the presence of a front and a back wall in the experimental box, as well as some variability due to the presence of defects. Other phenomenological differences between simulations and experiments are observed for the favored crystal orientation and slip events. We think that these differences are due to a small tilt of the experimental box and small variations of the experimental box width $L_{x}$.

For a system initialized as a perfect crystal, i.e., without initial defects, we consistently observe the wall-induced convective motion to occur at higher values of the dimensionless energy parameter (shaking strength) with respect to the system initialized randomly. From the analysis of the granular temperature we distinguish different regions based on the slope 
of the temperature as a function of the dimensionless energy $K_{m}$. For systems initialized randomly, i.e., with many initial defects, we distinguish two regions ( $A$ and $B$ ) of temperature. The transition between the two regions occurs at the onset of convection, but we do not observe any change in the slip probability in the transition between regions $A$ and $B$.

On the other hand, for systems initialized with a perfect hexagonal lattice, i.e., without initial defects, we distinguish three regions in the temperature curve. Moreover, in this case the slip probability has very different behavior in the three regions. In particular, in region $A_{1}$ the number of detected slips is exactly zero, while region $A_{2}$ is characterized by a quite steep increase of slip events with the input energy, which slows down considerably upon the onset of convection (region $B$ ).

The slip probability follows an Arrhenius law, indicating the presence of an activating mechanism. The very high barrier in the region $A_{2}$ of the system without defects is due to the nucleation of defects in the perfect hexagonal crystal. We noted that the barrier in region $B$ is the same for the system initialized with and without initial defects. In this region the behavior of the slip probability is related to the activation of slip events.

The results of our work provide an explanation for the onset of the convective regime in vertically oscillated granular systems and show that defects enhance the onset of convection, i.e., systems with topological defects show convection at lower oscillation strengths than systems without defects. More work is needed to quantify the degree of variability for the onset of convection due to its sensitivity to the concentration and possibly the types of defects. Interestingly, since defects can diffuse out of the system when they reach the top of the granular bed, regions of transient convective motion are possible, provided that the time scale for the defect diffusion is larger than the time scale for the nucleation of defects. This conjecture was not studied in this work, but represents an interesting avenue of future research. Furthermore, we plan to study how the size polydispersity of the granular particles changes the onset of the wall-induced convection as well as the influence of the box size on the dynamical behavior. Furthermore, we would like to explore the effect of shock waves [37] on the dynamical behavior of the system.

\section{ACKNOWLEDGMENTS}

The authors thank Ingo Rehberg and Matthias Schmidt for discussions and acknowledge Philipp Ramming for the help in image processing. Maximilian von Teuffenbach, Andreas Fischer, and Philip Krinninger are acknowledged for helping with the initial development of the granular simulation code. K.H. is supported by the Deutsche Forschungsgemeinschaft through Grant No. HU1939/2-1.

\section{APPENDIX A: DETAILS OF THE MODEL}

We carry out molecular dynamics simulations at fixed time step $d t$, for two translational degrees of freedom and one rotational degree of freedom for a system of soft disks. An illustration of the model is shown in Fig. 6. Two particles at positions $\boldsymbol{r}_{i}$ and $\boldsymbol{r}_{j}$ with velocities $\boldsymbol{v}_{i}$ and $\boldsymbol{v}_{j}$ and angular velocities $\boldsymbol{\omega}_{i}$ and $\boldsymbol{\omega}_{j}$ define a system with an effective mass $m_{\mathrm{eff}}=m_{i} m_{j} /\left(m_{i}+m_{j}\right)$ and a normal unit vector $\boldsymbol{n}_{i j}=$

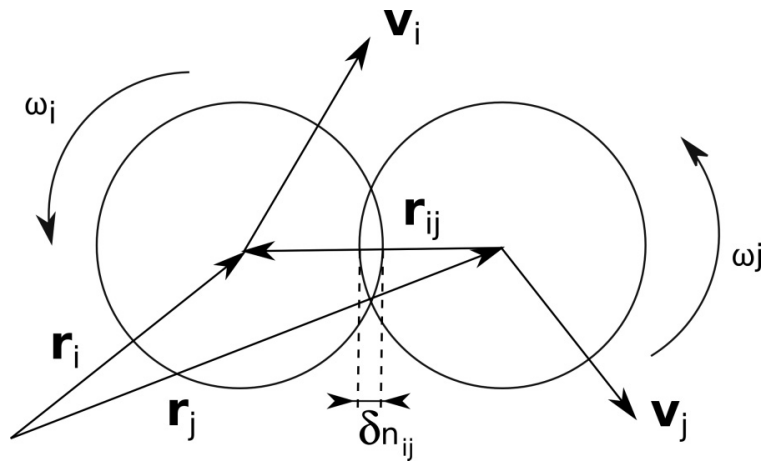

FIG. 6. Illustration of the contact model of two granular disks at distance $\boldsymbol{r}_{i j}=\boldsymbol{r}_{i}-\boldsymbol{r}_{j}$ and relative velocity $\boldsymbol{v}_{i j}=\boldsymbol{v}_{i}-\boldsymbol{v}_{j}$, which overlap by a distance $\delta_{n_{i j}}$.

$\frac{\boldsymbol{r}_{i}-\boldsymbol{r}_{j}}{\left|\boldsymbol{r}_{i}-\boldsymbol{r}_{j}\right|}$. The tangential direction is defined as $\boldsymbol{t}_{i j}=\frac{\boldsymbol{v}_{i_{j j}}}{\left|\boldsymbol{v}_{i j}\right|}$ where

$$
\boldsymbol{v}_{t_{i j}}=\boldsymbol{v}_{i j}-\boldsymbol{v}_{n_{i j}}-\frac{1}{2}\left(\sigma_{i} \boldsymbol{\omega}_{i}+\sigma_{j} \boldsymbol{\omega}_{j}\right) \times \boldsymbol{n}_{i j},
$$

with $\boldsymbol{v}_{n_{i j}}=\left(\boldsymbol{v}_{i j} \cdot \boldsymbol{n}_{i j}\right) \boldsymbol{n}_{i j}$.

We define the displacement in the two directions $\delta_{n_{i j}}=d-$ $\left|\boldsymbol{r}_{i}-\boldsymbol{r}_{j}\right|$, with $d=(1 / 2)\left(\sigma_{i}+\sigma_{j}\right)$, and $\delta_{t_{i j}} \boldsymbol{t}_{i j}=\boldsymbol{v}_{t_{i j}} d t$. For the disk-disk interactions we use a linear model with forces

$$
\begin{gathered}
\boldsymbol{F}_{n_{i j}}=\left(\kappa_{n} \delta_{n_{i j}} \boldsymbol{n}_{i j}-\gamma_{n} m_{\mathrm{eff}} \boldsymbol{v}_{n_{i j}}\right), \\
\boldsymbol{F}_{t_{i j}}=\left(-\kappa_{t} \delta_{t_{i j}} \boldsymbol{t}_{i j}-\gamma_{t} m_{\mathrm{eff}} \boldsymbol{v}_{t_{i j}}\right)
\end{gathered}
$$

in the normal and shear tangential directions, respectively. The parameters $\kappa_{n}$ and $\kappa_{t}$ are the stiffness coefficients in the normal and tangential directions, respectively. The energy dissipated during the contact is regulated by the damping coefficients $\gamma_{n}$ and $\gamma_{t}$. In addition we model the static friction by keeping track of the elastic shear displacement $\delta_{t_{i j}}$ over the contact lifetime and truncate it such that the Coulomb condition $\left|F_{t_{i j}}\right|<\left|\mu F_{n_{i j}}\right|$ is satisfied, where $\mu$ is the static friction coefficient.

The same kind of interaction is used between the particles and the container wall. We also consider the gravitational force $\boldsymbol{g}=-g \boldsymbol{e}_{z}$, where $\boldsymbol{e}_{z}$ is the unit vector pointing in the $z$ direction.

Once the forces on all particles are known the total force and torque $\tau_{i}$ on a particle $i$ is determined by

$$
\begin{gathered}
\boldsymbol{F}_{i}=m \boldsymbol{g}+\sum_{j}\left(\boldsymbol{F}_{n_{i j}}+\boldsymbol{F}_{t_{i j}}\right), \\
\boldsymbol{\tau}_{i}=-\frac{1}{2} \sum_{j} \sigma_{j} \boldsymbol{n}_{i j} \times \boldsymbol{F}_{t_{i j}} .
\end{gathered}
$$

The typical numerical values of the simulation parameters are shown in Table I. In this linear model it is possible to calculate the contact duration [29]

$$
t_{c}=\pi\left(\frac{k_{n}}{m_{\mathrm{eff}}}-\frac{\gamma_{n}^{2}}{4}\right)^{-0.5}
$$

In order to obtain an accurate integration of the equation of motion during contact, the time step of the simulation is chosen to be $\delta t \approx t_{c} / 50$ [38]. 


\section{APPENDIX B: DETECTION OF THE DYNAMICAL PHASES}

The detection of the convective motion, in both computer simulations and experiments, is performed by using $N_{t}$ tracer particles, which are initially positioned at the center of the oscillating box in a straight horizontal line. In order to determine the threshold for convection, we analyze the deviation of the imaginary line connecting the tracer particles from the initial straight horizontal configuration. This analysis is carried out by calculating, at the beginning of each cycle, the variance of the tracers' height from their average height,

$$
s_{j}^{2}=\frac{1}{N_{t}} \sum_{i=1}^{N_{t}}\left(z_{j}^{i}-\left\langle z_{j}\right\rangle\right)^{2},
$$

where $\left\langle z_{j}\right\rangle$ is the average vertical position of the tracers at frame $j$ and $z_{j}^{i}$ is the vertical position of tracer $i$ at the beginning of cycle $j$.

The average variance over all $N_{f}$ frames is calculated,

$$
s_{\mathrm{c}}^{2}=\frac{1}{N_{f}} \sum_{j=1}^{N_{f}} s_{j}^{2},
$$

and the start of the convection is identified via the condition $s_{\mathrm{c}}>\sigma$, where $\sigma$ is the diameter of the grains.

The detection of the bouncing bed dynamical phase is performed by detecting the detachment of the granular bed from the bottom plate, which can occur at any phase of the oscillating cycle. Therefore, we calculate the average height of the tracers with respect to the oscillating plate for each phase $k$,

$$
s(k)=\frac{1}{N_{f}} \sum_{j=1}^{N_{f}}\left[\left\langle z_{j}(k)\right\rangle_{i}-z_{\mathrm{b}}(k)\right],
$$

where $\left\langle z_{j}(k)\right\rangle_{i}=\frac{1}{N_{t}} \sum_{i=1}^{N_{t}} z_{j}^{i}(k)$ is the average height of all $N_{t}$ tracer particles in phase $k$ and cycle $j, z_{\mathrm{b}}(k)$ is the height of the plate in phase $k$, and $N_{f}$ is the total number of frames in a certain phase. Consequently, the variance over all $N_{k}$ phases is calculated:

$$
s_{\mathrm{b}}^{2}=\frac{1}{N_{k}} \sum_{k=1}^{N_{k}} s^{2}(k)
$$

and the bouncing bed phase is identified with the condition $s_{\mathrm{b}}>\sigma / 30$. The value $\sigma / 30$ corresponds to half a pixel of the experimental images. For the sake of comparison, the same value is used in the analysis of the simulation trajectories.
[1] H. M. Jaeger, S. R. Nagel, and R. P. Behringer, Rev. Mod. Phys. 68, 1259 (1996).

[2] S. Luding, Nonlinearity 22, R101 (2009).

[3] H. Miyamoto, H. Yano, D. J. Scheeres, S. Abe, O. Barnouin-Jha, A. F. Cheng, H. Demura, R. W. Gaskell, N. Hirata, M. Ishiguro, T. Michikami, A. M. Nakamura, R. Nakamura, J. Saito, and S. Sasaki, Science 316, 1011 (2007).

[4] J. Duran., Sands, Powders, and Grains: An Introduction to the Physics of Granular Materials (Springer-Verlag, Berlin, 2000).

[5] N. Mohabuth and N. Miles, Resour., Conserv. Recycl. 45, 60 (2005).

[6] A. Rosato, K. J. Strandburg, F. Prinz, and R. H. Swendsen, Phys. Rev. Lett. 58, 1038 (1987).

[7] M. Schröter, S. Ulrich, J. Kreft, J. B. Swift, and H. L. Swinney, Phys. Rev. E 74, 011307 (2006).

[8] D. C. Hong, P. V. Quinn, and S. Luding, Phys. Rev. Lett. 86, 3423 (2001).

[9] J. B. Knight, H. M. Jaeger, and S. R. Nagel, Phys. Rev. Lett. 70, 3728 (1993).

[10] W. Cooke, S. Warr, J. M. Huntley, and R. C. Ball, Phys. Rev. E 53, 2812 (1996).

[11] T. Pöschel and H. J. Herrmann, Europhys. Lett. 29, 123 (1995).

[12] A. Kudrolli, Rep. Prog. Phys. 67, 209 (2004).

[13] M. Majid and P. Walzel, Powder Technol. 192, 311 (2009).

[14] A. Mehta and J. M. Luck, Phys. Rev. Lett. 65, 393 (1990).

[15] S. Douady, S. Fauve, and C. Laroche, Europhys. Lett. 8, 621 (1989).
[16] A. Ugawa and O. Sano, J. Phys. Soc. Jpn. 72, 1390 (2003)

[17] O. Sano, Phys. Rev. E 72, 051302 (2005).

[18] P. Eshuis, R. Bos, D. Lohse, D. Van der Meer, and K. Van der Weele, Phys. Fluids 19, 123301 (2007).

[19] P. Eshuis, D. van der Meer, M. Alam, H. J. van Gerner, K. van der Weele, and D. Lohse, Phys. Rev. Lett. 104, 038001 (2010).

[20] J. Sun, F. Battaglia, and S. Subramaniam, Phys. Rev. E 74, 061307 (2006).

[21] E. Clemént, J. Duran, and J. Rajchenbach, Phys. Rev. Lett. 69, 1189 (1992).

[22] J. B. Knight, E. E. Ehrichs, V. Y. Kuperman, J. K. Flint, H. M. Jaeger, and S. R. Nagel, Phys. Rev. E 54, 5726 (1996).

[23] Y.-h. Taguchi, Phys. Rev. Lett. 69, 1367 (1992).

[24] S. Luding, E. Clément, A. Blumen, J. Rajchenbach, and J. Duran, Phys. Rev. E 50, R1762 (1994).

[25] M. Bourzutschky and J. Miller, Phys. Rev. Lett. 74, 2216 (1995).

[26] D. Risso, R. Soto, S. Godoy, and P. Cordero, Phys. Rev. E 72, 011305 (2005)

[27] D. Frenkel and B. Smit, Understanding Molecular Simulation (Academic Press, London, 2002).

[28] P. A. Cundall and O. D. L. Strack, Géotechnique 29, 47 (1979).

[29] J. Schäfer, S. Dippel, and D. E. Wolf, J. Phys. I 6, 5 (1996).

[30] H. K. Pak and R. P. Behringer, Phys. Rev. Lett. 71, 1832 (1993).

[31] This parameter is also called the dimensionless shaking strength [18]. 
[32] C. Kimme, D. H. Ballard, and J. Sklansky, Commun. ACM 18, 120 (1975).

[33] P. J. Steinhardt, D. R. Nelson, and M. Ronchetti, Phys. Rev. B 28, 784 (1983).

[34] V. W. de Villeneuve, R. P. Dullens, D. G. Aarts, E. Groeneveld, J. H. Scherff, W. K. Kegel, and H. N. Lekkerkerker, Science 309, 1231 (2005).
[35] F. Rietz and R. Stannarius, Phys. Rev. Lett. 108, 118001 (2012).

[36] We have chosen to exclude particles closer than $10 \sigma$ to the free interface.

[37] K. Huang, G. Miao, P. Zhang, Y. Yun, and R. Wei, Phys. Rev. E 73, 041302 (2006).

[38] J. Lee, J. Phys. A 27, L257 (1994). 